\title{
“V了”和 “VR”
}

周 刚

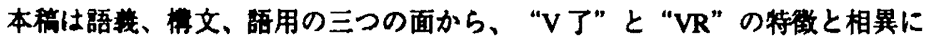
ついての分析、解噃を試みたものである。

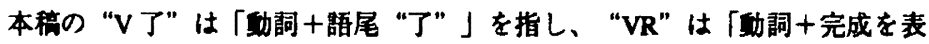
す結果補語」を指す。二者の意味はほほ同じであるが、具体的な用法は翼なる。 日本人の中国語学習者にはその区別が蜼しいと感じられる。

1. “V了”と“VR" の語数面の機能。

2. “了”と “R” のVに対しての㩧択。

3. “V了”と“VR" の槽文面の特鹳

4. “V了”と “VR" の語用面の相異。

0 前言

1. “V 了" 和 “VR”的语义功能

1.1 “V了" 的语义功能

1.2 "VR" 的语义功能

2. “了”和 “ $R$ ” 对 $V$ 的选择

2.1 “了”对 $V$ 的选择

2.2 “ $R$ ” 对 $V$ 的选择

\section{0 前言}

现代汉语中动词后加动态助词 “了”般表示动作和变化实现或完成，可以记作 “V了”：动词加某些结果补语包括某些表 结果的趋向动词, 表示动作变化完成, 象

$$
\begin{aligned}
& \text { “动十好”、“动十到”、“动十著”、 } \\
& \text { “动十上”、“动十下”、“动十起”等动 }
\end{aligned}
$$

补结构，可以记作 “VR”。“V了”和

“VR” 中的“了”和 “R” 由于意义十分相 似, 常常可以互换而不改变句子的基本意 思。诺着：

(1)a. 我㫓了饭, 穿了衣服, 就出去了。

b. 我吃好饭, 穿好衣服, 就出去了。
3. “V了” 和 “VR" 的句法特征

\section{1 前加否定状语}

3.2 前加时间状语

3.3 后带时旦宾语

3.4 中间插人 “得”和“不”

4. “V了”和 “VR" 在语用上的区别 4.1 动作和变化完成的强调程度不同

\section{2 表达的清晰度不同}

(2)a. 昨天她买了一件很漂亮的衣服。

b. 昨天她买到一件很漂高的衣服。

(3)a. 上星期天他钧了三条鱼。

b. 上星期天他钓语三条鱼。

(4)a. 去的时候, 商店已经关了门。

b. 去的时候，商店已经关上门。

(5)a. 快脱了衣服，烤一䞍。

b. 快脱下衣服、烤一烤。

(6)a. 村民们办了一所小学。

b. 村民们办起一所小学。

可是, 有时 “了”和 “R” 尽䈏意义相似， 却不能互换。例如 :

(7)a. 他去了三天。 


$$
\text { b.*他去好三天。 }
$$

(8)a. 小王跌了一交。

b.*小王跃着一交。

(9)a. 我没买上那本书。

b.*我没买了那本书。

日本学生对此常常感到难于区别。

汉语语法学界对 “V了" 和 “VR" 都有 过研究。对 “V 了”尤其对词尾 “了”比较 注意，研究成果甚多。但集中在 “了”的意 义方面 (1)。对 “VR" 的研究。还不够充 分, 只是主要对 “VR" 中的 $R$ 的虚化以及 "VR" 的否定式的否定范围等问题作了一 些探讨(2)。本文是在这些研究基础之上, 进 一步从语义、句法和语用三个平面对 “ $V$ 了”和 “VR”的语义功能、句法特征、语 用区别以及 “了”和 “ $R$ ”对 $V$ 的语义选择 限制等问题加以探讨和分析, 并作一些解 释。这些问题的探索不尽有助于对汉语动词 用法的了解, 而且对于汉语教学也具有应用 价值。

\section{1 "V了" 和 "VR" 的语义功能}

\section{1 “V 了" 的语义功能}

一般认为动态助词 “了”分两个了即 $了_{1}$, 和 $了_{2} ， 了_{1}$ 是词尾的 “了”，预是句尾 的 “了”。 “了”。（本文论述的 “了”，如无特别指 明，均指词尾的“了”。）它是由结果补语 发展而来的。“了”原来是动词“了”

（liăo），意思是 “完”，经过长期的演变过 程，“了”失去了原来的声调，变成轻声， 原来的词汇意义也逐渐消失, 虚化成为表示 语法意义的动态助词。 (3) “了" 的语法意义 一向认为是表示完成(4), 近年来有人认为是 表示实现(5), 也有人认为是实现或完成 (6) 其实词尾 “了”的语法意义不那么单纯。它 往往跟所粘附的动词本身的语义有密切关 系。

从动词所表示的动作或变化的时间特点 来看, 动词可以分成结束性动词和持续性动
词两大类。结束性动词表示的动作或变化是 瞬间完成的, 即动作的起点 (开始) 和终点 （完成）之间的时间非常短暂，甚至是重合 的。在句法形式上这类动词前边不能加 “正”、“正在”、“在”，后边不能加 “替”。例如常用的结束性动词有：

败超成到跌䅦见进陰 来免 (免职) 人伤死完忘陷 保证毕业 避免 承认出发 发现 感到 告诉 结束 开始 明白 批准 确定认识 失败 通知 同意习惯 原凉 允许 费成 知道 一些双音节的动补结构类似动词，都不能前 加“正”、“正在”、“在”，后加 “着”，可看作这一类动词，例如：

看见 听见 解开 分开 办成 画成 看出 提出 修完 学会 吃饱 说清 持续性动词表示的动作或变化都有一系列过 程，即有开始、持续、结束。在句法形式 上，这类动词前边可加“正”、“正在”、 “在”，后边也可以加 “兴”。持续性动词 数量很多, 先举几例 :

等看吃喝买拉查问考 洗 说 读表扬 改革 管理 广播 研究 学习整理 住 站 歇 爱

结束性动词后加 “了”，一般表示动作 或变化的完成。例如：

(10) 我情了很多东西。

(11) 我们承认了错误。

(12) 小王学会了开车。

持续性动词后加 “了”，有时表示动作 或变化的实现, 即动作或变化成为事实, 可 能刚开始，也可能正在进行。例如：

(13) 我住了新盖的楼房。

(14) 今年他学习了英语。

(13) 中的 “住” 这一动作可能刚开始. 也 可能正在进行。(14)亦然。有时又可表示动 作或变化已经结束。例如 :

（15）老师表扬了小张。

(15) 中的 “表扬” 这一动作已经结束。 


\section{2 “VR" 的语义功能}

"VR" 中的 $\mathbf{R}$ 是结果补语，可以由形 容词. 动词，也可以由趋向动词充当。但 是，这些词不同程度地虑化了，表现在本身 词汇意义正在逐步失去, 趋于表示引申意义 或某种语法意义，在语音上往往弱化念轻 声。下面分别谈谈这些词的语义功能。

“V+好”中的“好”，实义是表示完 尊。现在某些场合保留举实义用法。例如： “我们一定要学好汉语”。大部分 "V+ 好” 中的“好”失去了完善的意思，而表示 完成的意思。例如：

(17) 干好活儿再去吧。

(18) 你做好饭没有?

"V十到” 中的 “到”，实义是表示达 到, 涉及空间和时间概念。例如, “去年我 搬到东京”。“他活到 1975 年”。在用于 达到某种目的和结果, 意义就应化了。例 如：

(19) 我今天收到他的回信。

(20) 他已经做到这一点了。

“V十着”中的“着”，实义是表示附 着、现在这种意义使用得较少, 例如：“穿 着要整齐”。大部分 “ $\mathrm{V}$ 十着”中的 “举” 失去了附着的意思, 而表示动作达到了目 的。这时 “普”读轻声 “zhao"。

(21) 我买着汉语语法书了。

(22) 他打著两只鸟。

“ $v$ 十上”中的“上”，原是表示动作 方向, 例如, “大家一起走上楼”。现在用 于表示进人某种状态。达到目的或完成时。 就失去了趋向意义而虚化了。例如：

(23) 我和他交上朋友了。

(24) 快关上门。

这时 “上”念轻声。

“ $V+$ 十"中的“下”，原是表示动作 方向, 例如, “气车滑下山坡”。现在用于 表示完成时, 失去了趋向意义而虚化。例 如 :

(25) 我记下他的地址和电话号码。
（26）老王买下一套住房。 这时 “下”念轻声。

“ $\mathrm{V}$ 十起”中的“起”，原是表示动作 向上，例如，“他站起身，给老人坐”。现 在失去了'趋向意义，虚化为动作完成的意 义。例如：

（27）他们办起一家公司。

(28) 我收起笔记本，往外走去。 “ $v$ 十上”和 “ $v+$ 十”，有时意义比 较复杂，同一个动词加“上”或“下”，可 以表示两种意思, 即既可以有表示动作趋向 的实在意义, 也可以有表示结果的虚化意 义。比较 :

(29) 赶紧挂上奵笼。

（30）你真有办法，挂上她了。

(31) 扔下东西就走。

(32) 你不能扔下我不管。

(29）中“挂上”是指动作的趋向，(30)中 “挂上” 是指动作的结果，意思是 “和 她有了某种特殊关系”。(31)中“扔 下”是表示动作趋向。(32)中“扔下” 是表示动作结果。“上”、“下”的 虚、实义跟前边动词本身有密切的关 系。如果动词本身表示的动作具体实 在，而且有一定的方向性，后加的趋向 动词 “上”和 “下”意义也实在。如果 动词本身的意义不很实在，又不带一定 的方向性，后加的“上”和“下”也就 失去了实在的趋向义, 而虚化成结果 义。

有时，实在的趋向义和应化的结果义两 者兼备的情况也存在。例如：

（33）他终于穿上军装，成了一名祖国卫 士。

(33) 中 “穿上” 的 “上”，一方面具有动 作的趋向义, 军装往身上穿; 另一方面也具 有动作的结果义。终于穿了军装，实现了理 想。

“VR" 中 R 在句法结构中的语义表述 功能也趋于虚化。一般动补结构的语义表述 
重点往往在补语上。例如：

衣裳晾干了 $\rightarrow$ 衣裳干了

我听猫了 $\rightarrow$ 我猫了

他长大了 $\rightarrow$ 他大了

把动词拿去也能说。而 “VR" 中 $\mathbf{R}$ 的不是 语义表述重点, 不可以把动词全掉。请看：

我买到书 $\rightarrow *$ 我到书

小王关上窗 $\rightarrow *$ 小王上窗

我找着他了 $\rightarrow *$ *我举他了

一般动补结构中补语的语义指向往往有 这三种情况。

第一种、补语与主语或宾语有关。例

如 : ,

他喝醉了酒 $\rightarrow$ 他喝酒他醉了（与主语有 关)

他喝多了酒 $\rightarrow$ 他喝酒酒多了（与宾语有 关)

第二种。补语与主语和宾语都有关。例

如 :

玻缡被他打碎了一块 $\rightarrow$ 玻㻦碎了（与主 语有关）碎了一块（与宾语有关）

第三种、补语只与动词有关。例如：

他扯䋈我的衣服 $\rightarrow$ 紧扯（我的衣服）

这时补语具有说明功能。作用近似状语，一 般也可以移位作状语，说明动词表示动作、 变化的状态或状况。

“VR" 中 R 的完全没有上速前两种功 能, 只有第三种功能. 即与动词有关, 但是 由于已经虚化, 不能象一般动补结构中补语 可以移位作状语。例如：

他穿好衣服 $\rightarrow *$ 他好穿衣服

我买着一本书 $\rightarrow *$ 我曾买一本书

由此看来， R 跟一般补语不同，它的语 义表述功能也趋于虚化、而紧紧地粘附于动 词, 表示动态。

2 “了”和 “ $R$ ”对 $V$ 的选择

“V 了”和“VR”中“了”和“R”对 $V$ 的选择不同，具体地说 “了”和 “ $R$ ” 对 V 有语义选择限制，这种限制各不相同。
$2.1 “ 了 ”$ 对 $\mathrm{V}$ 的选择

V 跟“了”组合的语义选择限制宽松。 绝大部分动词都可以和“了”组合。只有少 数动词不可以跟“了”组合, 这些动词不表 示具体的动作和变化, 可称为无变化动词, 可记作 V(一变化)，例如，是、姓、如、 属、祝、比如、例如、等于、给以、加以、 好像、企图、认为、以为、主张等。

在一定的语境中，“姓”和“是”也可 以跟“了”组合、《中国语文》1991 年第4 期曾载文谈及这个问题，现在把例句抄下为 证。

（34）算大哥从那时就姓了䔬。

(35) “谁过继过?”二马说：“我从生 下来就姓了威，姓了二十多年了。

（36）辛楣虽然觉得这句话中听, 还不愿 意立刻放弃他的不快：“你这个人真没 有良心。吃了人家的饭，还要管闲事， 探听人家阴私。只要女人可以做太太, 管她什么美国人，俄国人。难道是了美 国人，她女人的成分就加了倍? 养孩子 的效率会与众不同？"

一般说来，可以和 “了”组合的动词是 表示具体的动作和变化的动词，可以记作 V (十变化)。

\section{(2.2) $R$ 对 $V$ 的选择}

$\mathrm{V}$ 跟 R 组合，语义限制较为严格。从总 的方面来看, 可以跟 $R$ 组合的 $\mathrm{V}$, 首先, 一 般是表示具体的动作和变化的动词，即 $V$ 〔十变化〕。不表示具体的动作和变化的动 词即 V(一变化)，不能跟R组合。比较：

$\begin{array}{ll}\text { 写好信寄走 } & \text { * 姓好王 } \\ \text { 打著一只鸟 } & \text { *等于着十 } \\ \text { 养上两头牛 } & \text { * 如上小王 } \\ \text { 造起三层楼 } & \text { *像起他 }\end{array}$

其次，表示具体的动作和变化的动词不 是全部都可以跟 $\mathbf{R}$ 组合，再必须是其中的持 续性动词，才可以跟 R 组合。这些动词可记 作 V (十变化) (十持续)。结束性动词即 $V$ [十变化) (一持续) 不可以跟 R 组合。 
比较:

$\begin{array}{ll}\text { 看好电影 } & \text { * 散好会 } \\ \text { 买到东西 } & \text { * 猫到知识 } \\ \text { 考上学校 } & \text { *伯上困难 } \\ \text { 收起行本 } & \text { * 断起念头 }\end{array}$

再次, 也不是全部 V(十变化)(十持 续了，还必须是其中表示动作者有意识地发 出的动作、行为的动词即自主动词(7), 才可 以跟 $\mathbf{R}$ 组合，这些动词可以记作 V ? +变 化 )（十持续）（十自主）。其中的非自主 动词即 $V($ 变化)(十持续)(一自 主了，不可以跟 $\mathbf{R}$ 组合。比较：

$\begin{array}{ll}\text { 做好饭 } & \text { * 跌好跤 } \\ \text { 找前目标 } & \text { *迷蓄路 } \\ \text { 创下奇迹 } & \text { *忘下事情 } \\ \text { 闭起眼 } & \text { *闪起腰 }\end{array}$

第四，V(十变化 $、$ 十持续 $〔 十$ 自 主了可以跟 $R$ 组合, 是指九许组合的一般语 义限制，在此范围内，具体情况还要视 $\mathbf{R}$ 的 个别词而定。

V 跟“好”组合面相对最宽，常用的动 词可跟表完成义的“好”组合的, 有以下这 些(8)。

看 瞧 听吃喝 吐喷吸抽

吹偿尝取抓捏握摸

捞 找寻摘抹揉拍揓

刻卷揭解安放挏提举

推拉扯拖牵运托搬拔

搭 捧铺摆扶打站砍摇

插硅扔接投撤采捆绑

编开张关闭分包贯称

盛装拉埋堵填按貼压

挂 拣拨拾拣招交接跑

跳 踩 踢 停坐躺伸 䪙 喼

跃解钻靠挤拧扭绞㳦

躲背带歇睡穿脱臷披

织染煡补裁浸洗晒刷

是级梳悌刮擦拈烧

炒㘼蒸意繁泡炸腌杀

割利剔切喂住留盖锁
酒扫洗谈讲念唱叫㖑 骂 哭 笑 闹 劝理问约用 尽借送寄派发做弄干 㩦管办造钉锯铲丵唐 碾繁弹㷧种栽钩买卖 使 付 业算换选评罚使 查抱乘 骑 划收教学读 考 试抄 涂 画 玩想记数 加添演钓莘雇捋搜莹 奖 端 垒

观化荠 打扮收拾 交代 访问 邀请 交涉 费助介绍 招待 庆祝 啗咐 委托 接受 收割计算 统计 核对 清理 合作 保护动员 推广 开展 宣传 攱动 说明 解释 补充 汇报 报告 发表 举行 参加 讨论 谣论 争论 表决总结 商量协商 联合 组合 登记 集中 表扬 批评 批判检讨 评论反省修改发扬 改变 利用 使用 准备 搜集 实行 照顾 调查 安排 争吵 通知 训练 培菲 练习研究 银炼了解建设 考虑 回忆解剖 劳动 组织

常用的动词可跟表示达到某种目的和结果 的“到”组合的, 有以下这些。

\begin{tabular}{|c|c|c|c|c|c|c|c|c|}
\hline & 䁃 & 㕵 & 㷡 & 闻 & 见 & 吃 & 喝 & 吸 \\
\hline & 惯 & 吸 & 抽 & 吹 & 㗍 & 尝 & 亲 & 吻 \\
\hline & 取 & 抓 & 捏 & 握 & 摸 & 捞 & 找 & \\
\hline & 抹 & 䒨 & 揉 & 姜 & 拍 & 拱 & 揭 & \\
\hline & 拉 & 扯 & 拖 & 牵 & 托 & 搬 & 拔 & \\
\hline & 夹 & 抱 & 搂 & 打 & 敲 & 扯 & 欣 & \\
\hline & 投 & 拐 & 采 & 分 & 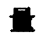 & 称 & 盛 & \\
\hline & 挖 & 掘 & 点 & 压 & 圭 & 挑 & 拾 & \\
\hline & 传 & 接 & 踩 & 踢 & 踹 & 跸 & 追 & \\
\hline & 桷 & 挤 & 补 & 费 & 剃 & 刮 & 榇 & \\
\hline & 炸 & 割 & 槑 & 切 & 勒 & 住 & 酒 & \\
\hline & 讲 & 念 & 叫 & 喊 & 鹏 & 问 & 约 & \\
\hline & 用 & 尽 & 借 & 送 & 邮 & 捎 & 寄 & \\
\hline & 荧 & 夸 & 作 & 弄 & $\mp$ & 嘱 & 管 & \\
\hline & 钟 & 賏 & 弹 & 㷧 & 锄 & 买 & 孯 & \\
\hline
\end{tabular}


算换选评罚使查数 骗

哄混偷抱夺乘骑划收

学读考抄涂画演想猜

数 钓 搜

参观 招呼 访问 邀请 迎接介绍 委托 收割 计算 统计 核对 清理 动员 补充 參加 讨论 争取 联合 影响 利用 使用 照顾 安排 通知 研究了解留心考虑

常用的动词可跟表示达到目的和结果的 “着”组合的，就是上逨可跟“到”组合的 这些单音节动词，不再另列。

常用的动词可跟表示达到目的、进入某 种状态或完成的“上”组合的, 有以下这 些。

看瞧 见吃喝咬喷吸抽

吹㗍尝拿取抓摸捞找

寻抹㨲操搓拍班刻卷

安放搁提举推拉拖牵

运托搬搭捧捏铺摆 夹

抱 搂 打 就 欧 插 投拐 采

栶 邨 编 开关闭分合包

量称盛装接埋堵填岸

贴 絬 压挂挑拨拣招交

走 跑 跳 蹦 踩 踢 踏 站 立

跟爬闯撞坐躺跑喼动

翻钻追靠挤打背带歇

睡穿裁披织染继补裁

浸洗 晒刷㻏量梳擦烧

炒烤蒸妋墨整泡炸腌

切喂 住盖锁酒扫说谈

讲念喝 叫喊䴗哭笑闹

劝问约等呆待陪求用

借送捎寄派交发惯惹

夸爱养做弄干据管办

造佟钉锯铲丵唐碾轧

䢃 弹 炼种栽 买久付絮

赔 算 换 选 评 使 卟 哄混

抱 乘 骑 收 端 教 学 读考

抄涂画玩恨佳气记想
猜 数 加 添 演 劝钓 雇

查

参观 休息 打扮 治疗 招呼 邀请 帮助 招待 夸奖 统计 核对 清理 合作 拥护 动员 参加 联合 争取 表扬 批评 利用 使用 照顾 调查 安排 通知 留心 关心

常用的动词可跟表示完成的“下”组合 的, 有以下这些。

吃喝吞咽咬啨吸抽余

取摘揭 解 放 㨄 扯拖拔

铺 摆 打 砍括碰扔接投

甩采扎垶卸挖埋压掘

拔传交接停闯藏脱睡

呆待剃剔割刺切住留

酒夸做搞办造了种栽

买赎 欠换罚救夺收发

记抄 立

常用的动词可跟表示完成的“起”组合 的, 有以下这些。

安坚插㧢 绑编 开关

包衰盛装挖掘贴挂立

穿裁披复梳剃住盖漏

办造修种栽救想起钓

收

收拾 动员 联合 组织 训练 培养 建设

总之, $\mathbf{R}$ 选择语义特征 (十变化)〔十 持续〕[十自主〕的动词组合。 $R$ 的个别具 体词组合面各有不同，其中“好”和“上” 对 V 选择最宽，“到”和“畕”次之，

“下”和“起”则最严。这可能是更深层次 的语义特征制约羔这种选择，值得作进一步 的探讨。

还有一个值得注意的现象是, $\mathrm{R}$ 所选择 的动词大多数是单音节动词, 只有一小部分 是双音节动词, 而且这些双音节动词是日常 生活中最常用的。因此 “VR” 具有口语性 强的特点。 
3 “V 了" 和 “VR" 的句法特征

"V了" 和 "VR" 的语义都表示动作或 变化的动态, 二者存在一些细微差别, 这些 差别可以在句法结构上反映出来。“v了” 和 “VR” 的句法特征的差异主要有以下几 点。

3.1 前加否定状语

(一) 前加“没有”

“V了”前边不可以加“没 (有) ”。 “VR" 则可以。比较：

*没 (有) 吃了饭

没（有）吃好饭

*没 (有) 找了小王

没（有）找蓄小王

*没(有) 穿了衣服

没 (有) 穿上衣服

“V 了”的否定式不是“没 (有) $\mathrm{V}$ $\mathrm{J}^{\prime}$ ”，而是“没 (有) $\mathrm{V}$ ”，是指动作或变 化没有成为事实。“VR" 的否定式是没

(有) VR" , 是指动作或变化没有完成。 前者否定动作或变化的本身，例如 “吃?” 的否定式是 “没吃”，意思不是没吃完。而 是根本没有吃的动作 : 后者是否定动作或变 化的完成或结果, 例如 “吃好”的否定式是 “没（有）吃好”，意思不是没有吃。而是 正在吃，但还没有吃完。

\section{(二) 前加 “不”}

“V了" 和 “VR" 前边都可加 “不”， 但是必须在一定的语境中才可以。换言之, “不V了十名”和“不 VR+名”都不能独立 成句, 只能作为条件分句, 后边还需要加上 其他成分才行。请看：

*不吃了饭/不吃了饭, 再走吗?

*不找了小王/不找了小王, 一齐去 吗?

*不吃好饭/不吃好饭，不许走。 *不找著小王/不找首小王, 不罢 休。

后边的成分常常以反问句或者否定的结果分 句形式出现。

3.2 前加时间状语

(一) 前加 “将 (要)

"VT" 的前边不可加 “将 (要) " (9).
"VR" 的前边则可以加。比较：

*将（要）吃了饭将（要）吃好饭

*将 (要) 穿了新衣 将 (要) 穿上新衣 * 将 (要) 盖了楼房将 (要) 盖起楼房

"V了" 表示动作或变化已经实现或完成。 成为一种事实。如果用表示将来的时间副词 去伤饰 “v 了”，就完全矛盾了。这在情理 上讲不通。例如，“盖了楼房，可真不容 易。”说这句话的时候，楼房已经盖好了, 还要用 “将 (要) “去佟䇥 “盖了楼房”。 那就不行。因此 “V 了”前边不可加“将 (要) "。“VR" 表示动作、变化的结 果, 不涉及是否成为事实, 也就是说这种结 果可以预测。例如, “盖起楼房, 可真不容 易。”说这句话的时候，也许楼房正在盖， 还没盖好。因此 “VR” 前边可以加表示将 来的时间副词。例如可以说 “这儿将要盖起 楼房”。

(二) 前加 “已经”

"v了" 可以前加 “已经”。例如：

已经看了电影

已经买了衣服

已经学了汉语

“VR”的前边也可以加“已经”，不 过一般还要在 “VR”的后边加“了!”。或 者在句末加上 “了 2 ”。否则会觉得有点第 不住. 感到别扭。比较:

?已经看好电影 已经看好了电影 已经晋好电影了

? 已经买到衣服 已经买到了衣服 已经买到衣服了

? 已经学上汉语 已经学上了汉语 已经学上汉语了

其原因可能跟 “VR" 不涉及动作是否成为 事实有关。前加 “已经”，其意思是指动作 或变化已成为事实, 因此“VR”后边还要 加表示成为事实的“了”，或者在句末加 “了 ${ }_{2} "$. “了 2 " 既表示事物或状态出现了 变化, 又表示一种确定的语气, 而且它还有 成句的作用。 
3.3 后带时 量宾语

“V ?" 和 “VR" 后带时兵宾语有以下 两种情况。

\section{（一）只带时量宾语}

“V了"可以带时是兵语。“VR" 后带 时量宾语时，则不能单独成句。比较：
看了三天
*看好三天
买了三天
*买䈐三天
挂了三天
*挂上三天
插了三天
*插下三天

“V了”中的 V 是持续性动词(10, 带上 时量宾语后，有些是表示动作行为本身的持 续时旦，例如“看了三天”，“买了三 天”。有些是既可以表示动作行为本身的持 续时量，又可以表示动作完成后形成状态的 持续时间，例如“挂了三天”，“括了三 天” 价。“VR”中的 V 虽然本身是持续性 动词, 但是加上 $\mathbf{R}$ 以后, 由于前边不能加 “正”、“正在”、“在”，后边不能加 “羔”，就有了结束性动词的特点。这时时 量宾语就只指动作完成之后经历的时间, 而 且不能单独成句, 必须后加一些成分才行。 例如 :

(37) 书看好三天了。

(38) 才买著三天就坏了。

(39) 刚挂上三天又摘下来了。

\section{（二）带“时量宾语十了”}

“V了”和“VR”都可以带“时量宾语 十了”，而且也都可以单独成句。例如:

$\begin{array}{ll}\text { 买了一年了 } & \text { 买着一年了 } \\ \text { 查了两年了 } & \text { 查好两年了 } \\ \text { 挂了半个月了 } & \text { 挂上半个月了 } \\ \text { 插了半个月了 } & \text { 插下半个月了 }\end{array}$
但是二者表示的意思不尽相同。“V 了十时 量宾语十了”的语义比较复杂，不同的动词 类，含义也不同。象“查”、“买”一类, 带“时量宾语十了”时, 可以有两种意思, 一种是动作行为到说话时, 持续了一段时 间，而且还在继续: 还有一种急思是动作行 为完成以后到说话时.已经历了一段时间。
例如 :

买了一年了，还没有买啚（持续）

买了一年了, 还是挺新的 (完成)

查了两年了，还没有查好（持续）

查了两年了, 又该査了（完成）

象“挂”、“括”一类，带“时量兵语十 了”时，可以有三种意思。第一种急思是动 作行为到说话时，持续了一段时间，还在继 续：第二种意思是动作行为完成以后到说话 时，已经历了一段时间：第三种意思是动作 行为完成以后造成的状态到说话时，已持续 了一段时间。例如：

挂了半年了，还没有挂好 (动作持续) 你挂了半天了，早歇过来了，又该挂了 (完成)

标语挂了半年了，得摘下来了（状态持 续)

“VR十时量宾语十了”的语义比较简 单。“查”、“买”一类动词组成的 “VR”，带“时量宾语十了”只有一种意 思. 即指动作行为完成以后到说话时, 已经 历了一段时间。例如:

查好两年了, 还有一年时间，再查一 次

买下两年了, 还挺新的

“挂”、“插”一类动词构成的“VR”， 带“时量宾语十了”，则有两种意思，一种 是动作行为完成以后到说话时, 已经历了一 段时间：另一种是动作行为完成以后造成的 状态到说话时，已持绕了一段时间。例如：

那块木牌括下半天了。这块怎么还没插 (完成)

指示牌插下三年了。该换新的了（状态持 续)

3.4 中间插人 “得” 和 “不”

“V了”中间不能括人 “得”和“不”

(2)。“VR”中间则能插人 “得”和 “不”，表示可能义。例如：

吃得好吃不好

买得著买不者 


$\begin{array}{ll}\text { 自得到 } & \text { 看不到 } \\ \text { 关得上 } & \text { 关不上 } \\ \text { 脱得下 } & \text { 脱不下 } \\ \text { 办得起 } & \text { 办不起 }\end{array}$

由此可见，“V 了”结合较紧密。 “VR”的结构则比较松散，虽然已经应 化, 但是还没有成为动态助词, 仍然充当补 语。

\section{4 “V了”和 “VR”在用语上的区别} “V了”和 “VR" 既使在意义和用法一 致的场合下, 即可以互换, 急思基本不变的 情况下，二者在语用上也存在某些差别。

4.1 对动作或变化的完成的强调程度不 同

在贸调动作或变化的完成的程度上. “V $"$ 不及 “VR"。尤其是憵调动作或变 化的完成具有某种不寻常的意义时，多用 “VR"。比较 :

(40) a.今天是小王的生日, 他高兴地穿 上一件新衣服。

b. 今天是小王的生日, 他高兴地穿 了一件新衣服。

（41） a. 她去了好几次，才买着那本书。

b. 她去了好几次，才买了那本书。

(42) a. 经过一番努力, 他终于独立做好 这项工作。

b. 经过一番努力, 他终于独立做了 这项工作。

(40)中 a. 比 b. 更强调为庆祝生日而穿新衣 服的总义。(41)中 a. 比 b. 更强调买书的 不易。(42)中 a. 比 b. 更强调独立工作的 结局。

\section{2 表述的清晰度不同}

说话者要使听话者正确地理解话语内 容, 应选择表达清晰度高的格式, 即选择意 义准确、简洁而没有歧义的格式。“v 比“VR”容易产生歧义。例如、“学了三 天了”这一格式具有“一直学了三天”和 “学结束三天了”两种意思。如果是表述后
一种急思，就应选择“学好三天了”这一种 格式，可见“V了”不如 “VR”表达清晰。

〈注〉

(1)近期的主要有吕叔湘 1980 , 刘月华等 1983, 刘勋宁 1988, 本英哲 1990 , 房玉 清 1992 等论著。

(2) 见房玉清 1992, 内田度市 1981 , 相原 茂 1979 等论著。

(3)启无消 1992第十五章

(4)例如赵元任: A Grammar of Spoken Chinese. 王力：《中国语法理论》，吕叔 湘朱德熙:《语法信辞讲话》都把词尾 “了”的语法怠义解程为 “完成”

(5)刘勋宁 1988。

(6)同 (3)

(7)急见马庆株 1992

(B)常用动词主要选自《普通话三千常用词 表》，郑林睡等编，文字改革出版社、 1987 和《常用字和常用词》, 北京语言学 院语言数学研究所编, 北京语言学院出版 社. 1985。

(9)将要V了“(例如 “将要吃了”, 将要做 了”) 的“了”是“了 ${ }^{\prime}$, 即句尾 "了"。

(10)结束性动词已可以进人 “ $v$ 了十时是宾 语” 这一格式, 但是因与 VR 比较, 故不 提及。

(11)同(7)

(2) “V 得了”和“V 不了”（例如“吃得 了”和“吃不了”) 的“了” (liăo) 是 动词, 不是动态助词“了”（le）。

\section{〈主要参考文献〉}

房玉消：1992,《实用汉语语法》, 北京语 言学院出版社。

马庆株：1992, 《汉语动词和动词性结 构》, 北京语言学院出版社。 李英哲等：1990，《实用汉语參考语法》悲 文华译, 北京语言学院出版社。 
刘勋宁：1988, 《现代汉语词尾 “了”的语 法意义》，《中国语文》1988 年第 5 期 321-330 页。

刘月华等：1983，《实用现代汉语语法》。 外语教学与研究出版社。

吕权湘主编：1980,《现代汉语八百词》, 商务印书馆。
内田市：1981。「結果補語と否定の射 程, ‘中国語研究 20, , 龍涣吾舍, 21-31 页。

相原茂：1979，「否定を拒む二・三の要 因」。'中国語研究 19, , 能溪害舍, 31-32 页。 